\title{
Determination of temperature dependent parameters of zero-phonon line in photo-luminescence spectrum of silicon-vacancy centre in CVD diamond thin films
}

\author{
Kateřina Dragounová, ${ }^{*},{ }^{* *}$ Zdeněk Potůček, ${ }^{*}$ \\ Štěpán Potocký, ${ }^{* *}$ Zdeněk Bryknar, ${ }^{*}$ Alexander Kromka ${ }^{* *}$
}

\begin{abstract}
In this work we present a methodological approach to the temperature dependence of photoluminescence (PL) emission spectra of the silicon-vacancy centre in diamond thin films prepared by chemical vapour deposition. The PL spectra were measured in the temperature range of $11-300 \mathrm{~K}$ and used to determine the temperature dependence of the zero-phononline full-width at half-maximum and of the peak position. Experimental data were fitted by models of lattice contraction, quadratic electron-phonon coupling, homogeneous and inhomogeneous broadening. We found that the shift of peak position and peak broadening reflect polynomial dependence on temperature. Moreover, a proper setting of monochromator slits width is discussed with respect to line profile broadening.
\end{abstract}

K e y w or d s: silicon-vacancy centres, photoluminescence, low temperature, diamond, CVD

\section{Introduction}

Diamond shows a unique combination of physical and chemical properties such as high hardness, thermal conductivity, chemical stability, wide band gap $(\sim 5.5 \mathrm{eV})$ $[1,2]$ and biocompatibility, which make it advantageous for optoelectronics [3,4] and biotechnology [5]. These applications are determined not only by the intrinsic properties of diamond but also by crystallographic defects and impurities. Considerable attention is recently devoted to the creation of colour centres with potential uses as solid state light emitters for quantum optics [6], photonics [7] and biomedicine [5]. Such uses require controllable fabrication of structures (single diamond crystals, nanorods, periodic structures, etc.) [8-10]. Negatively charged silicon-vacancy $(\mathrm{SiV})$ centres in diamond belong to the group of colour centres in diamond that are prospective for photonic applications as single photon sources and fluorescent markers for bio-imaging. The SiV photoluminescence (PL) spectra consist of the dominant, sharp zerophonon line (ZPL) in the vicinity of $738 \mathrm{~nm}$ at room temperature and practically invisible phonon sidebands. A highly photostable SiV centre consists of a substitutional silicon atom incorporated between two vacant lattice positions forming the so-called split-vacancy configuration [11]. A near infrared SiV PL exhibits a short lifetime of $1.2 \mathrm{~ns}$ [6]. The importance of $\mathrm{SiV}$ centres study arises from common silicon contamination in diamonds prepared by the microwave plasma enhanced chemical vapour deposition (CVD) process due to the etching of Si substrates, quartz windows or bell jars [12].

Recently, luminescence properties of SiV centre in diamond thin films and nanoparticles have been widely investigated. The dependence of $\mathrm{SiV}$ photoluminescence activity on the deposition parameters has been systematically investigated [13-16]. It has been found that the substrate material quality has no effect on $\mathrm{SiV}$ [4] and PL is sensitive to the gas composition [14] and substrate temperature [15]. Optimal gas composition and substrate temperature resulting in maximal PL activity were reported in [16]. To identify the origin of SiV centre electronic transitions, structure and geometry, ab initio calculations [17], EPR measurements and study of their optical properties in polarized light $[11,12,18]$ were performed. Subsequently, a model of SiV centre energy levels within the band gap was proposed. Neu et al [19] ascribed temperature dependence of ZPL parameters observed in the low-stress homo-epitaxial CVD diamond thin film to the influence of lattice contraction and electron-phonon interactions in the perturbed lattice. This idea was partly supported by Jahnke et al [20], who also took into consideration the effect of spin-orbital interaction.

To contribute to this discussion, we present a methodological approach how to measure and analyze the temperature dependent photoluminescence emission spectra of $\mathrm{SiV}$ centres in diamond thin films prepared by the microwave plasma assisted CVD process. The suggested ap-

\footnotetext{
Department of Solid State Engineering, Faculty of Nuclear Sciences and Physical Engineering, Czech Technical University in Prague, Trojanova 13, 12000 Prague, Czech Republic, katerina.dragounova@fjfi.cvut.cz

** Department of Optical Materials, Institute of Physics, Academy of Sciences of the Czech Republic, Cukrovarnická 10 , 162 00 Prague, Czech Republic
} 


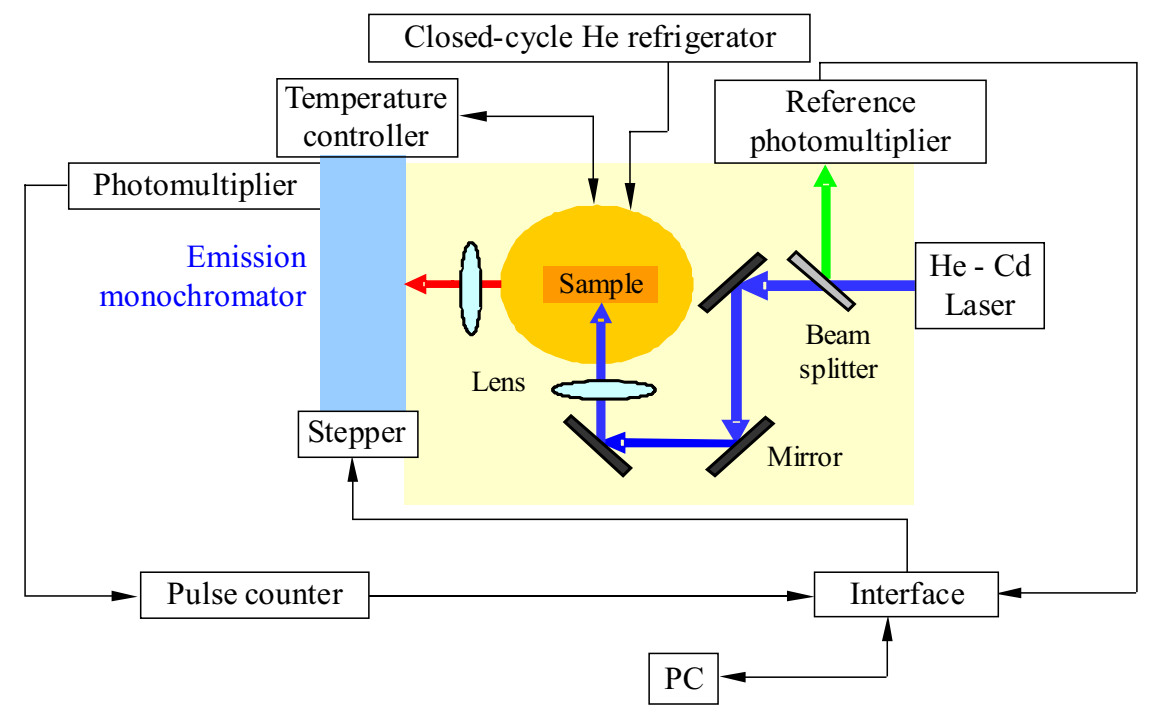

Fig. 1. A schematic view of the experimental setup used for measurement of temperature dependent photoluminescence emission spectra

proach is affirmed by comparing experimentally obtained results with proposed theoretical models.

\section{Experimental}

Diamond thin film was grown on a quartz substrate with a dimension of $10 \times 10 \mathrm{~mm}^{2}$. Before the CVD growth, the substrate was ultrasonically pre-treated in a suspension of deionised water and ultra-dispersed detonation diamond powder (diameter 5-10 nm, New Metals and Chemicals Corp. Ltd.). The CVD deposition of diamond was performed in a microwave plasma enhanced CVD reactor with an ellipsoidal resonator using focused hydrogen-rich plasma with methane $\left(1 \% \mathrm{CH}_{4}\right.$ in $\mathrm{H}_{2}$ ), [21]. During the diamond deposition, no siliconcontaining gas mixtures were used.

Temperature-dependent PL emission spectra of the diamond thin film excited by $442 \mathrm{~nm}$ line of $\mathrm{He}-\mathrm{Cd}$ laser were measured in the nearly right angle geometry within the 11 to $300 \mathrm{~K}$ temperature range using a setup based on a Carl Zeiss SPM2 monochromator (grating 650 grooves/mm, spectral range 193 to $1022 \mathrm{~nm}$ ). The PL intensity was measured with a cooled RCA31034 photomultiplier (GaAs photo-cathode, spectral range 200 to $930 \mathrm{~nm}$ ) operating in the photon-counting mode. The intensity of incident excitation light was monitored by the photomultiplier 67 PK511 (photocathode S20) behind the cuvette with a solution of rhodamine B in ethylene glycol. All the presented emission spectra were corrected for the spectral dependence of the apparatus response. Figure 1 schematically shows the setup of the optical measurement system.

\section{Results and disscusion}

\section{Photoluminescence spectra}

We paid attention to the selection of a sufficiently small width of emission monochromator slits to ensure minimal influence of line profile broadening by the monochromator. This allows us to determine the temperature development of the ZPL parameters (in particular FWHM). This procedure is mainly important in the lowtemperature region, where the narrowest ZPL FWHM is expected. The spectral line broadening by the monochromator can be omitted if the monochromator bandpass, influenced by slits width, is much smaller than the line FWHM [25].

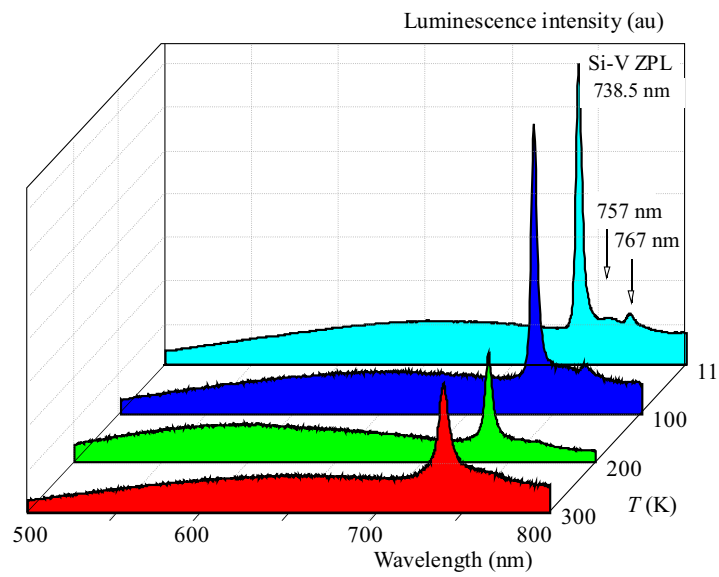

Fig. 2. Temperature dependent photoluminescence emission spectra of diamond thin film (with dominant ZPL at $738.5 \mathrm{~nm}$ ) excited by $442 \mathrm{~nm}$ line of He-Cd laser

In other cases, the measured shape of the emission spectrum does not represent/reflect the real spectrum and the influence of instrumental line profile must be 

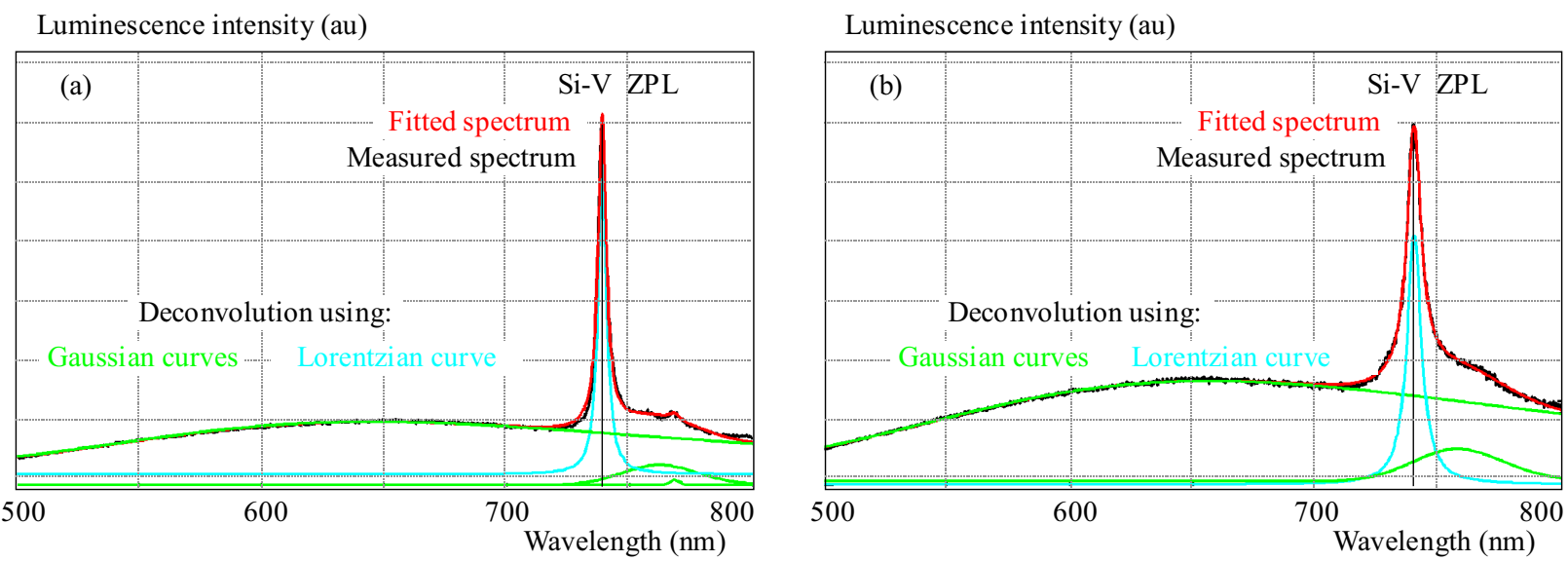

Fig. 3. Examples of typical spectrum decomposition for two temperature regions (below and above $200 \mathrm{~K}$ ) of SiV centre photoluminescence in a diamond thin film under excitation of $442 \mathrm{~nm}$ line of He-Cd laser. (a) - low-temperature region (150 K) fitted by one Lorentzian and three Gaussian curves, (b) — room-temperature emission spectrum fitted by one Lorentzian and two Gaussian curves.
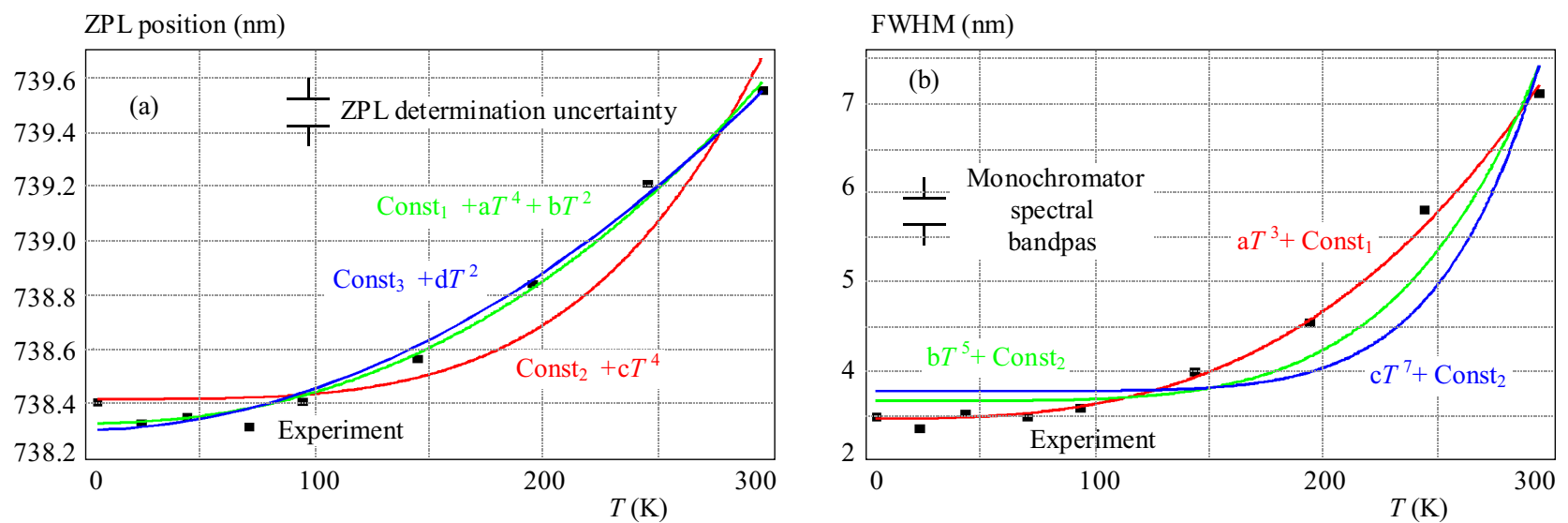

Fig. 4. Temperature development of (a) peak position and (b) half-width (FWHM) obtained for SiV centre ZPL in a diamond thin film under excitation by $442 \mathrm{~nm}$ line of He-Cd laser: (a) - uncertainty in the determination of ZPL position is depicted in figure, and (b) the monochromator spectral bandpass

taken into account. For our measurement we used a slit width of $0.13 \mathrm{~mm}$ corresponding to the monochromator bandpass of $0.52 \mathrm{~nm}$, which is more than 6 -times lower than the ZPL full width at half maximum of SiV centre emission.

We assume that ZPL broadening is a combination of two contributions - inhomogeneous broadening resulting from the presence of impurity centres in the perturbed host lattice and homogeneous broadening arising from the electron-phonon coupling [19]. The latter is a temperature dependent and characterized by a Lorentz line profile. Contrariwise, the former should be temperature independent and described by a Gauss line profile. Thus, we proposed to fit the shape of our measured emission ZPL by a Lorentzian curve and considering inhomogeneous broadening as an invariable contribution. Taking into account the origin of the broadband with a maximum at $650 \mathrm{~nm}$ and ZPL phonon sidebands, we fitted PL spectra with one Lorentzian curve and three Gaussian curves for temperatures up to $200 \mathrm{~K}$ to subtract the nonlinear ZPL background. For higher temperatures, where the peak at $757 \mathrm{~nm}$ became less pronounced, the same fit quality was achieved by using one Lorentzian curve and only two Gaussian curves. Representative examples of spectrum decomposition are shown in Fig. 3 .

The temperature dependence of ZPL position and FWHM are shown in Fig. 4. The figures show experimental data fitted with polynomial curves according to the discussed models. A blue shift and narrowing of ZPL with temperature decrease is clearly observed. Pronounced changes take place primarily in the region from room temperature down to $100 \mathrm{~K}$ where the peak position shifts to lower wavelengths (higher energies) by $1.2 \mathrm{~nm}$ and FWHM decreases from $7 \mathrm{~nm}$ to $3.5 \mathrm{~nm}$. Further decreasing of temperature down to $11 \mathrm{~K}$ did not significantly contributed to the ZPL position and FWHM changes which remain nearly constant.

\section{Lorentzian and two Gaussian curves}

Variation of the ZPL peak position with temperature could be explained as a result of two synergetic pro- 
cesses corresponding to lattice contraction and quadratic electron-phonon coupling $[19,20]$. These processes can be described by the dependence $a T^{4}+b T^{2}$ where $a$ and $b$ are constants. The first term $a T^{4}$ represents the effect of mutually inseparable contributions of lattice contraction and electron-phonon coupling with hard phonon modes. The second term $b T^{2}$ emerges additionally in the expression when the softening of bonds in the centre excited state is also taken into account. Very good agreement of this model with our experimental data is shown in Fig. 4(a) where the dominance of $T^{2}$ terms is shown. Neu et al reported that for $\mathrm{SiV}$ colour centres, the temperature dependence of ZPL FWHM could be described by three terms proportional to $T^{7}, T^{3}$, and $T^{5}$, [19]. Hence, we fitted experimental data in Fig. 4(b) with functions linearly dependent on $T^{7}, T^{5}$, and $T^{3}$ and looked for the best match. The accuracies of our fits were $86.0 \%$ for term $T^{7}, 93.6 \%$ for $T^{5}$ and $99.5 \%$ for $T^{3}$. It is apparent that the best fit was obtained for $T^{3}$ dependence.

The $T^{7}$ term corresponds to the influence of phonons of a perturbed crystal structure on the transition between non-degenerate electron states within Debye approximation. The sum of the first and second terms, $T^{7}$ and $T^{3}$, reflects the impact of electron-phonon coupling in the case of degenerate electronic states including the effect of softening of bonds in the colour centre excited state. At the same time, the dominance of the $T^{3}$ term was verified for $\mathrm{N}-\mathrm{V}$ centre [26]. The third term $T^{5}$ is characteristic for the temperature dependence of the ZPL width under the action of dynamic Jahn-Teller effect in the involved degenerate electronic state. The $T^{3}$ dependence was also ascribed to the effect of fluctuating fields resulting from modulation of the distances between SiV centre and other defects in the film due to their interaction with phonons. It was concluded that the dominant term in this expression determines the main mechanism of the FWHM broadening. This dependence is most probably a consequence of defects in the structure of diamond film [19].

\section{Conclusions}

We introduce an experimental methodology for studying the temperature behaviour of silicon-vacancy centre photoluminescence in diamond thin films. The correctness of the used approach was demonstrated by measurement and analysis of photoluminescence emission spectra in the temperature range from $11 \mathrm{~K}$ to $300 \mathrm{~K}$. The ZPL peak position blue shift for decreasing PL temperature was described by $a T^{2}+b T^{4}$ dependence and explained by lattice contraction and weakening of the strength of quadratic electron-phonon coupling. The ZPL narrowing with decreasing temperature was best described by the $T^{3}$ dependence. This behaviour was explained by homogeneous broadening determined mainly by fluctuating fields between the SiV centre and other defects in the film in consequence of their interaction with phonons.

\section{Acknowledgements}

This work was supported by the grant No. SGS16/ 244/OHK4/3T/14 of the Grant Agency of the Czech Technical University in Prague, by grant No. 14-04790S (KD, SP) of the Czech Science Foundation and by the grant of MEYS No. RVO14000.

\section{REFERENCES}

[1] T. Brodić,"Trends in developing new semiconductor power devices based on $\mathrm{SiC}$ and diamond materials", Journal of Electrical Engineering, vol. 52, No. 34, 2001, pp. 105-116.

[2] C. E. Nebel," Semiconductor materials: From gemstone to semiconductor", Nat Mater, vol. 2, No. 7, 2003, pp. 431-432.

[3] R. U. A. Khan, B. L. Cann, P. M. Martineau, J. Samartseva, J. J. P. Freeth, S. J. Sibley, C. B. Hartland, M. E. Newton, H. K. Dhillon, and D. J. Twitchen," Colour-causing defects and their related optoelectronic transitions in single crystal CVD diamond", Journal of Physics. Condensed Matter: An Institute of Physics Journal, vol. 25, No. 27, 2013, pp. 275801.

[4] M. Marton, M. Mikolášek, J. Bruncko, I. Novotný, T. Ižák, M. Vojs, H. Kozak, M. Varga, A. Artemenko, and A. Kromka," Fabrication and Characterization of N-Type Zinc Oxide/P-Type Boron Doped Diamond Heterojunction", Journal of Electrical Engineering, vol. 66, No. 5, 2015,.

[5] T. D. Merson, S. Castelletto, I. Aharonovich, A. Turbic, T. J. Kilpatrick and A. M. Turnley," Nanodiamonds with silicon vacancy defects for nontoxic photostable fluorescent labeling of neural precursor cells", Optics Letters, vol. 38, No. 20, 2013, pp. 4170.

[6] M. Leifgen, T. Schröder, F. Gädeke, R. Riemann, V. Métillon, E. Neu, C. Hepp, C. Arend, C. Becher, K. Lauritsen, and O. Benson," Evaluation of nitrogen- and silicon-vacancy defect centres as single photon sources in quantum key distribution", New Journal of Physics, vol. 16, No. 2, 2014, pp. 23021.

[7] S. Singh, V. Thomas, D. Martyshkin, V. Kozlovskaya, E. Kharlampieva and S. A. Catledge," Spatially controlled fabrication of a bright fluorescent nanodiamond-array with enhanced far-red Si-V luminescence", Nanotechnology, vol. 25, No. 4, 2014, pp. 45302.

[8] T. Ižák, M. Domonkos, O. Babchenko, M. Varga, B. Rezek, V. Jurka, K. Hruška and A. Kromka," Technological Aspects in Fabrication of Micro- and Nano-Sized Carbon Based Features: Nanorods", Periodical Arrays and Self-Standing Membranes, Journal of Electrical Engineering, vol. 66, No. 5, 2015,

[9] A. M. Berhane, S. Choi, H. Kato, T. Makino, N. Mizuochi, S. Yamasaki, and I. Aharonovich," Electrical excitation of silicon-vacancy centers in single crystal diamond", Applied Physics Letters, vol. 106, No. 17, 2015, pp. 171102.

10] C. Wang, C. Kurtsiefer, H. Weinfurter and B. Burchard," Single photon emission from SiV centres in diamond produced by ion implantation", Journal of Physics B: Atomic, Molecular and Optical Physics, vol. 39, No. 1, 2006, pp. 3741.

[11] U. F. S. D'Haenens-Johansson, A. M. Edmonds, B. L. Green, M. E. Newton, G.Davies, P. M. Martineau, R. U. A. Khan and D. J. Twitchen," Optical properties of the neutral silicon split-vacancy center in diamond", Physical Review, vol. B 84, No. 24, 2011,.

12] E. Neu, R. Albrecht, M. Fischer, S. Gsell, M. Schreck and C. Becher," Electronic transitions of single silicon vacancy centers in the near-infrared spectral region", Physical Review, vol. B 85, No. 24, 2012.

[13] V. S. Sedov, I. I. Vlasov, V. G. Ralchenko, A. A. Khomich, V. I. Konov, A. G. Fabbri and G. Conte," Gas-phase growth of 
silicon-doped luminescent diamond films and isolated nanocrystals", Bulletin of the Lebedev Physics Institute, vol. 38, No. 10, 2011, pp. 291-296.

[14] I. Sakaguchi, M. Nishitani-Gamo, K. P. Loh, H. Haneda, S. Hishita and T. Ando," Silicon incorporation into chemical vapor deposition diamond: A role of oxygen", Applied Physics Letters, vol. 71, No. 5, 1997, pp. 629.

[15] S. Singh and S. A. Catledge," Silicon vacancy color center photoluminescence enhancement in nanodiamond particles by isolated substitutional nitrogen on 100 surfaces", Journal of Applied Physics, vol. 113, No. 4, 2013, pp. 44701.

[16] Š. Potocký, T. Ižák, M. Varga and A. Kromka," Influence of gas chemistry on Si-V color centers in diamond films", physica status solidi (b), vol. 252, No. 11, 2015, pp. 2580-2584.

[17] A. Gali and J. R. Maze," Ab initio study of the split silicon-vacancy defect in diamond: Electronic structure and related properties", Physical Review, vol. 88, No. 23, pp. 2013,.

[18] L. J. Rogers, K. D. Jahnke, M. W. Doherty, A. Dietrich, L. P. McGuinness, C. Müller, T. Teraji, H. Sumiya, J. Isoya, N. B. Manson and F. Jelezko" Electronic structure of the negatively charged silicon-vacancy center in diamond", Physical Review, vol. 89, No. 23, 2014,.

[19] E. Neu, C. Hepp, M. Hauschild, S. Gsell, M. Fischer, H. Sternschulte, D. Steinmüller-Nethl, M. Schreck and C. Becher," Low-temperature investigations of single silicon vacancy colour centres in diamond", New Journal of Physics, vol. 15, No. 4, 2013, pp. 43005.

[20] K. D. Jahnke, A. Sipahigil, J. M. Binder, M. W. Doherty, M. Metsch, L. J. Rogers, N. B. Manson, M. D. Lukin and F. Jelezko," Electronphonon processes of the silicon-vacancy centre in diamond", New Journal of Physics, vol. 17, No. 4, 2015, pp. 43011.

[21] M. Füner, C. Wild and P. Koidl," Novel microwave plasma reactor for diamond synthesis", Applied Physics Letters, vol. 72, No. 10, 1998, pp. 1149-1151.

[22] A. Dietrich, K. D. Jahnke, J. M. Binder, T. Teraji, J. Isoya, L. J. Rogers and F. Jelezko," Isotopically varying spectral features of silicon-vacancy in diamond", New Journal of Physics, vol. 16, No. 11, 2014, pp. 113019.

[23] L. Bergman,, M. T. McClure, J. T. Glass and R. J. Nemanich,” The origin of the broadband luminescence and the effect of nitrogen doping on the optical properties of diamond films", Journal of Applied Physics, vol. 76, No. 5, 1994, pp. 3020.

[24] K. Iakoubovskii, G. J. Adriaenssens and M. Nesladek," Photochromism of vacancy-related centres in diamond", Journal of Physics: Condensed Matter, vol. 15, No. 2, 2000, pp. 189-199.

[25] I. Valenta and J. Pelant," Luminiscenční spektroskopie 1.- Objemové krystalické polovodiče", Vyd. 1. Praha, Academia, 2006, pp. 328, (in Czech).

[26] V. Hizhnyakov, H. Kaasik and I. Sildos," Zero-Phonon Lines: The Effect of a Strong Softening of Elastic Springs in the Excited State", physica status solidi, vol. (b) 234, No. 2, 2002, pp. 644-653.
Received 7 July 2016

Kateřina Dragounová (MSc), received her MSc degree in solid state engineering from the Czech Technical University in Prague in 2012. She has been a PhD student at the Department of Solid State Engineering of the Faculty of Nuclear Sciences and Physical Engineering of the Czech Technical University in Prague. Her research interests are focused on photoluminescence of impurities in diamond thin films and perovskite-type compounds.

Štěpán Potocký (MSc, PhD), graduated in mathematical and physical engineering in 2001 and received his $\mathrm{PhD}$ in plasma physics and physics of thin films in 2006, both from the University of West Bohemia, Pilsen. He is engaged in CVD and PVD technology, wide band gap semiconductors and lowtemperature plasma characterization.

Alexander Kromka (MSc, PhD, DrSc), received his MSc degree in electronic engineering from the Slovak University of Technology, Bratislava in 1995. He had been a PhD student at the Department of Microelectronics of the Faculty of Electrical Engineering and Information Technology and received the $\mathrm{PhD}$ degree in 2001. In 2016, he received the DrSc degree from the Slovak Academy of Sciences. Since 2009, he has been a group and project leader at the Institute of Physics, Czech Academy of Sciences. His research interests include diamond thin growth phenomena, low-temperature plasma processes, fabrication of advanced electronic and optical diamond devices, developing of artificial substrates for tissue engineering and regenerative medicine.

Zdeněk Bryknar (MSc, PhD, Prof), graduated from the Czech Technical University in Prague, Faculty of Nuclear Sciences and Physical Engineering, in 1967 and received here $\mathrm{PhD}$ in solid state physics in 1976. His research activity is devoted to optical properties of solids (bulk and thin films, $e g$, ferroelectric crystals and ceramics, mercurous halides, and tungstates) to clear up the formation of point defects and electronic structure of impurities in these materials and determination of their influence on crystal properties.

Zdeněk Potůček (PhD), graduated in 1994 and received $\mathrm{PhD}$ in 2001, both in physical engineering, from the Faculty of Nuclear Sciences and Physical Engineering, Czech Technical University in Prague. His research activity is focused on optical spectroscopy of intrinsic point defects and impurities in dielectric and semiconductor materials interaction of metals with polymers, atomic force microscopy and other surface analytical methods. 